\title{
Safety of Vaccines Used for Routine Immunization in the United States \\ Executive Summary
}

\section{Background}

Vaccines are considered one of the greatest public health achievements of the last century for their role in eradicating smallpox and controlling polio, measles, rubella, and other infectious diseases in the United States. ${ }^{1}$ Despite their effectiveness in preventing and eradicating disease, substantial gaps in vaccine uptake persist. Vaccination rates for young children are high; ${ }^{2}$ however, vaccination rates remain well below established Healthy People 2020 targets for many vaccines recommended for adolescents, ${ }^{3}$ adults, ${ }^{4}$ and pregnant women. ${ }^{5}$

In the United States, vaccine guidelines are set by the Centers for Disease Control and Prevention's Advisory Committee for Immunization Practices (ACIP). The number of routine immunizations recommended for children and adolescents, adults, and pregnant women has expanded considerably over the past 10 years. For example, since 2005, the routine adolescent vaccination schedule has grown to include the following vaccines at ages 11 or 12 years: meningococcal conjugate vaccine; tetanus, diphtheria, and acellular pertussis (Tdap); human papillomavirus (HPV); and influenza (one dose annually). Tables A-C display ACIP recommendations as of October 2011. These recommendations were in effect when this review began.

\section{Evidence-based Practice Program}

The Agency for Healthcare Research and Quality (AHRQ), through its Evidencebased Practice Centers (EPCs), sponsors the development of evidence reports and technology assessments to assist publicand private-sector organizations in their efforts to improve the quality of health care in the United States. The reports and assessments provide organizations with comprehensive, science-based information on common, costly medical conditions and new health care technologies. The EPCs systematically review the relevant scientific literature on topics assigned to them by AHRQ and conduct additional analyses when appropriate prior to developing their reports and assessments.

AHRQ expects that the EPC evidence reports and technology assessments will inform individual health plans, providers, and purchasers as well as the health care system as a whole by providing important information to help improve health care quality.

The full report and this summary are available at www.ahrol.gov/research/ findlings/evidence-based-reports/ ptsafetyuptp.html.

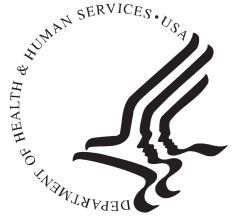


Table A. Vaccines routinely recommended for children and adolescents, 2011

\begin{tabular}{|c|c|}
\hline Vaccine & Age \\
\hline DTaP (diphtheria, tetanus, and acellular pertussis) & 2 months -6 years \\
\hline Hepatitis A & 12 months and older \\
\hline Hepatitis B & Birth and older \\
\hline Hib (Haemophilus influenzae type b) & 6 weeks -59 months \\
\hline HPV (human papillomavirus) & $\begin{array}{l}9 \text { years-21 years (male) } \\
9 \text { years-26 years (female) }\end{array}$ \\
\hline Influenza (inactivated) & 6 months and older \\
\hline Influenza (live attenuated) & 2 years and older \\
\hline IPV (inactivated polio vaccine) & 6 weeks and older \\
\hline MCV (meningococcal conjugate vaccine) & 2 years and older \\
\hline MMR (measles, mumps, and rubella) & 12 months and older \\
\hline MPSV (meningococcal polysaccharide vaccine) & 2 years and older in specific circumstances \\
\hline PCV13 (pneumococcal conjugate vaccine) & 6 weeks-18 years \\
\hline Pneumococcal polysaccharide vaccine & 2 years and older in specific circumstances \\
\hline Rotavirus & 6 weeks -8 months \\
\hline Tdap (tetanus, diphtheria, and acellular pertussis) & 7 years and older \\
\hline Varicella & 12 months and older \\
\hline
\end{tabular}

Table B. Vaccines routinely recommended for nonpregnant adults, 2011

\begin{tabular}{|c|c|}
\hline Vaccine & Recommendation \\
\hline Hepatitis A & All adults at increased risk for hepatitis A infection \\
\hline Hepatitis B & $\begin{array}{l}\text { All unvaccinated adults at risk for or requesting protection from } \\
\text { hepatitis B infection }\end{array}$ \\
\hline HPV (human papillomavirus) & Adults 26 years and younger \\
\hline Influenza (inactivated) & All adults \\
\hline Influenza (live attenuated) & All adults 49 years and younger \\
\hline $\begin{array}{l}\text { Meningococcal conjugate vaccine (MCV4) and meningococcal } \\
\text { polysaccharide vaccine (MPSV) }\end{array}$ & $\begin{array}{l}\text { Adults at risk of meningococcal disease (MCV4 or MPSV if } \\
\text { younger than } 55 \text { years; MPSV if } 55 \text { years and older) }\end{array}$ \\
\hline MMR (measles, mumps, and rubella) & All adults \\
\hline Pneumococcal polysaccharide vaccine & $\begin{array}{l}\text { Adults } 64 \text { years and younger with certain conditions and all } \\
\text { adults } 65 \text { years and older }\end{array}$ \\
\hline Td (tetanus, diphtheria) & All adults who are not immune through prior infection \\
\hline Tdap (tetanus, diphtheria, and acellular pertussis) & All adults 19-64 years old; some adults 65 years and older \\
\hline Varicella & All adults without evidence of varicella immunity \\
\hline Zoster & All adults 60 years and older \\
\hline
\end{tabular}

an 2013, pregnant women were advised to receive Tdap during every pregnancy to protect their newborns from pertussis. 
Table C. Vaccines routinely recommended for pregnant women, 2011

\begin{tabular}{|l|l|}
\hline Vaccine & Recommendation \\
\hline Hepatitis B & Recommended in some circumstances \\
\hline Influenza (inactivated) & All pregnant women \\
\hline Td (tetanus, diphtheria) & If indicated \\
\hline Tdap (tetanus, diphtheria, and acellular pertussis) & All pregnant women at the first trimester if indicateda \\
\hline
\end{tabular}

As the number of recommended immunizations has expanded across the population, so too have concerns about the safety of vaccines. Perhaps the most highly publicized safety concern of the last two decades was the proposed link between autism and the measles, mumps, and rubella (MMR) vaccine, first reported in 1998 in The Lancet by Dr. Andrew Wakefield. ${ }^{6}$ In 2010, The Lancet fully retracted the 1998 report, ${ }^{7}$ noting that elements of the research had been deliberately falsified. Although multiple large studies have confirmed the lack of association between MMR and autism, parental worries about the safety of vaccines persist. Other parental concerns about childhood vaccines include potential links to multiple sclerosis, sudden infant death syndrome, asthma, and diabetes. ${ }^{8}$ Thus, vaccine safety is high on the Nation's public health agenda.

\section{Objectives}

The Agency for Healthcare Research and Quality (AHRQ) requested an evidence report on the safety of vaccines recommended for routine immunization of adults (including pregnant women), children, and adolescents as of October 2011. This report, which represents the results of a comprehensive and systematic review of scientific evidence, describes potential associations between vaccines and adverse events (AEs) and will be used by the Office of the Assistant Secretary for Health (OASH) to identify the gaps in evidence. The report was guided by the following Key Questions (KQs):

KQ 1 . What is the evidence that vaccines included in the 2011 immunization schedule recommended for U.S. adults $^{9}$ are safe in the short term (within 30 to 42 days following immunization) or long term ( $>42$ days after immunization)?

a. What adverse events are collected in clinical studies (Phases I-IV) and in observational studies containing a control/comparison group? b. What AEs are reported in clinical studies (Phases IIV) and in observational studies containing a control/ comparison group?

c. What AEs are associated with these vaccines?

1. For each $\mathrm{AE}$ associated with a particular vaccine, what is the average severity (grade 1/mild; grade $2 /$ moderate; grades 3 and 4/severe)?

2. For AEs without statistically significant associations with a particular vaccine, what is the level of certainty?

3. For each $\mathrm{AE}$ associated with a particular vaccine, what are the risk factors for the AE (including age, sex, race/ethnicity, genotype, underlying medical condition, whether a vaccine is administered individually or in a combination vaccine product, schedule of vaccine administration, adjuvants, and medications administered concomitantly)?

KQ 2. What is the evidence that vaccines included in the immunization schedules recommended for U.S. children and adolescents in 201110 are safe in the short term (within 30 to 42 days following immunization) or long term ( $>42$ days after immunization)?

a. What AEs are collected in clinical studies (Phases IIV) and in observational studies containing a control/ comparison group?

b. What AEs are reported in clinical studies (Phases IIV) and in observational studies containing a control/ comparison group?

c. What AEs are associated with these vaccines?

1. For each $\mathrm{AE}$ associated with a particular vaccine, what is the average severity (grade 1/mild; grade 2/ moderate; grades 3 and 4/severe)?

a Level of certainty was operationalized as the 95-percent confidence interval surrounding the risk or odds estimate-i.e., the statistical significance 
2. For AEs without statistically significant associations with a particular vaccine, what is the level of certainty?a

3. For each $\mathrm{AE}$ associated with a particular vaccine, what are the risk factors for the AE (including age, sex, race/ethnicity, genotype, underlying medical condition, whether a vaccine is administered individually or in a combination vaccine product, schedule of vaccine administration, adjuvants, and medications administered concomitantly)?

KQ 3. What is the evidence that vaccines recommended for pregnant women ${ }^{11}$ are safe both for the woman and for her fetus/infant?

a. What AEs are collected in clinical studies (Phases IIV) and in observational studies containing a control/ comparison group?

b. What AEs are reported in clinical studies (Phases IIV) and in observational studies containing a control/ comparison group?

c. What AEs are associated with these vaccines in women?

1. For each $\mathrm{AE}$ associated with a particular vaccine, what is the average severity (grade 1/mild; grade $2 /$ moderate; grades 3 and 4/severe)?

2. For AEs without statistically significant associations with a particular vaccine, what is the level of certainty?a

3. For each $\mathrm{AE}$ associated with a particular vaccine, what are the risk factors for the AE (including age, sex, race/ethnicity, genotype, underlying medical condition, whether the vaccine is administered individually or in a combination vaccine product, the schedule of vaccine administration, adjuvants, and medications administered concomitantly)?

d. What AEs are associated with these vaccines in the fetus/infant?

1. For each $\mathrm{AE}$ associated with a particular vaccine, what is the average severity (grade 1/mild; grade 2/ moderate; grades 3 and $4 /$ severe)?

2. For AEs without statistically significant associations with a particular vaccine, what is the level of certainty?a

3. For each AE associated with a particular vaccine, what are the risk factors for the $\mathrm{AE}$ (including age, sex, race/ethnicity, genotype, underlying medical condition, whether the vaccine is administered individually or in a combination vaccine product, the schedule of vaccine administration, adjuvants, and medications administered concomitantly)?

\section{Methods}

In 2011, the Institute of Medicine (IOM) published a consensus report titled "Adverse Effects of Vaccines: Evidence and Causality." 12 That report evaluated the scientific evidence for event-vaccine relationships and covered the following vaccines on the 2011 recommended immunization schedules: varicella, influenza, hepatitis A, hepatitis B, HPV, MMR, meningococcal, tetanus, diphtheria, and pertussis. We report the IOM findings and update them by identifying and evaluating studies published after the IOM searches. We also searched for studies of pneumococcal, rotavirus, Haemophilus influenzae type $b$, inactivated poliovirus, and zoster vaccines; these were not included in the IOM report.

We searched electronic databases such as Medline ${ }^{\circledR}$ for relevant studies; complete search terms are provided in Appendix A of the full report. Databases were searched from inception through August 2013 for the vaccines not covered by the IOM report; for the other vaccines, the searches dated from a year before the IOM search. We also reviewed ACIP statements, package inserts, and Scientific Information Packets requested from vaccine manufacturers by an AHRQ-funded Scientific Resource Center. Finally, we scanned review articles for relevant references.

The following study designs were included:

- Controlled clinical trial. Human subjects are assigned prospectively, usually through randomization, to receive an intervention (in this case, a vaccine) or an alternative intervention (another vaccine) or placebo. Clinical trials are used to determine safety and efficacy. ${ }^{13}$

- Cohort study. Cohort studies follow two or more similar groups that differ with respect to whether they received a vaccine (the "exposure") to determine how/ whether the vaccination affects rates of one or more AEs (the "outcome"). 13,14

- Case-control study. Case-control studies compare people who have a disease or adverse event ("cases") with people who do not have the disease or event ("controls") and look back retrospectively to compare exposure to vaccine in each group to determine the relationship between the vaccine and the disease/ event. ${ }^{13,14}$

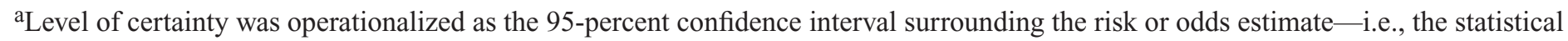
significance 
- Self-controlled case series (SCCS). Only cases (individuals who experienced the $\mathrm{AE}$ ) are included in the analysis. Each individual serves as his or her own control. The analysis inherently controls for covariates that remain stable within a person during the study period - for example, race and sex. SCCSs compare outcome event rates during times when a person is exposed (postvaccination) with those during times when the same person is unexposed (prevaccination) to calculate the relative incidence of AEs. ${ }^{13,15}$

- Other designs. We included all active surveillance studies that used regression to control for confounders and test multiple relationships simultaneously. We refer to these as multivariate risk factor analyses. Data sources may include medical records, health insurance claims, and government registries. ${ }^{13}$

Studies that use passive surveillance, such as the U.S. Vaccine Adverse Event Reporting System, ${ }^{16}$ are crucial in identifying signals regarding AEs postlicensure. However, because by definition they do not consider the rate of such events in nonvaccinated populations, they are not designed to assess a statistical association between a vaccine and an adverse event, so such studies were excluded from this project. We also excluded studies of vaccine formulations never used or no longer available in the United States. Examples include whole-cell pertussis vaccine, oral polio vaccine, and PCV7 pneumococcal vaccine. The recent IOM report "The Childhood Immunization Schedule and Safety: Stakeholder Concerns, Scientific Evidence, and Future Studies" 17 makes recommendations for future research on childhood vaccination schedules and cumulative effect, so this report focuses on assessing the association between AEs and specific vaccines rather than the cumulative effect of vaccines.

Two researchers independently reviewed the titles and abstracts identified. The union of their selections was retrieved. Two researchers also independently reviewed the full text of study reports and met to reach consensus regarding exclusion/inclusion. Disputes were settled by the lead investigators and team physician experts. Patient and study characteristics were abstracted by single researchers and confirmed by the principal investigator.

If a study reported severity or if adequate information was provided for our investigators to categorize severity, we used the Common Terminology Criteria for Adverse Events classification system 18 to characterize AEs. The definition of "serious" differs by AE type; each category of AE (e.g., fever, headache) is rated on a scale of 1 to 5 , with 1 being very mild and 5 being death due to the event.
The McHarm instrument ${ }^{19}$ was used to evaluate the quality of the studies with regard to assessment of adverse events. Studies that reported timing and severity and that defined AEs using standard precise definitions were rated higher than those that did not. (Many studies provided data on a list of AEs but did not address severity.) Epidemiological studies that used medical records to ascertain vaccination and health outcomes were rated higher than those that relied on patient or parent report.

We assessed the overall strength of evidence using guidance suggested by AHRQ for its Effective Health Care Program.20 This method is based on one developed by the GRADE (Grading of Recommendations Assessment, Development and Evaluation) Working Group ${ }^{21}$ and classifies the evidence according to the following criteria:

- High. High confidence that the evidence reflects the true effect. Further research is very unlikely to change our confidence in the estimate of effect.

- Moderate. Moderate confidence that the evidence reflects the true effect. Further research may change our confidence in the estimate of effect and may change the estimate.

- Low. Low confidence that the evidence reflects the true effect. Further research is likely to change our confidence in the estimate of effect and is likely to change the estimate.

- Insufficient. Evidence either is unavailable or does not permit a conclusion.

The evidence grade is based on four primary (required) domains and four optional domains. The required domains are risk of bias, consistency, directness, and precision, as described in the Methods section of the full report. The additional domains are dose response, plausible confounders that would decrease the observed effect, strength of association, and publication bias.

It is important to note that the 2011 IOM report used different terminology; evidence was classified as either "convincingly supports," "favors acceptance," "inadequate to accept or reject," or "favors rejection" of a causal association. The IOM included mechanistic studies and individual case reports to assess the biological plausibility of AEs and considered this information in addition to any statistical association. For each vaccine discussed in the IOM report, we started with the IOM findings and modified them, if needed, based on any additional evidence identified. If the IOM found that evidence "convincingly supports" an association, we rated the strength of evidence as "high" unless additional evidence was identified. Similarly, if the IOM found evidence "favors acceptance" 
we started with by rating as "moderate" strength of evidence and evidence rated as "inadequate to accept or reject" was considered "insufficient" in our grading system. If new evidence was identified for vaccines evaluated by the IOM, ratings could be adjusted up or down according to our assessment of the new studies. If the IOM found that evidence "favors rejection" of a causal relationship, we chose between moderate and high based on our review of the IOM evidence plus any studies published after their search.

\section{Results}

As presented in Figure A, a total of 20,478 titles were identified through electronic literature searches; review of product inserts; review of Food and Drug Administration, ACIP, and other Web sites; reference mining; and requests for Scientific Information Packets from drug manufacturers. Of those, 17,270 were excluded upon review of abstract or title, mostly due to lack of data on safety of vaccines. Other reasons for exclusion included use of vaccines not within the scope of this project (e.g., not formulations available in the United States, recommended only for travel), publication in languages other than English, and study not conducted on humans. Five reports could not be obtained; based on their titles, we do not think this affected the project.

Based on abstract screening, 3,210 articles were selected for full-text review. Of those, 392 were identified as relevant background/theoretical materials and set aside as potential references. A total of 2,650 other articles were excluded. The most common reason for exclusion was lack of suitable study design $(1,549)$ : individual case reports, nonsystematic reviews, and studies using passive surveillance were excluded. Many publications (458) discussed vaccines on the recommended schedule but did not report or assess AEs.

Studies using formulations never available or discontinued in the United States were excluded at full-text review (e.g., $\mathrm{H} 5 \mathrm{~N} 1$ vaccine, vaccines with the squalene adjuvant ASO3, and BCG vaccine against tuberculosis). Determining whether the potency or formulation was the one approved for clinical use in the United States was often difficult; the process involved comparing the potency, dosage, and ingredients listed on product materials and in Food and Drug Administration filings with the information reported in the study.

Based on full-text screening, 166 studies were accepted for abstraction. These include 86 controlled trials or cohort studies directly comparing a group that received a vaccine with an unvaccinated group. (Five of the 86 studies also reported a multivariate analysis.) We also abstracted 80 case-control studies, SCCSs, or multivariate risk factor analyses that met our inclusion criteria. These studies are in addition to those included in the 2011 IOM consensus report "Adverse Effects of Vaccines: Evidence and Causality," which were not abstracted. 
Figure A. Study/literature flow diagram

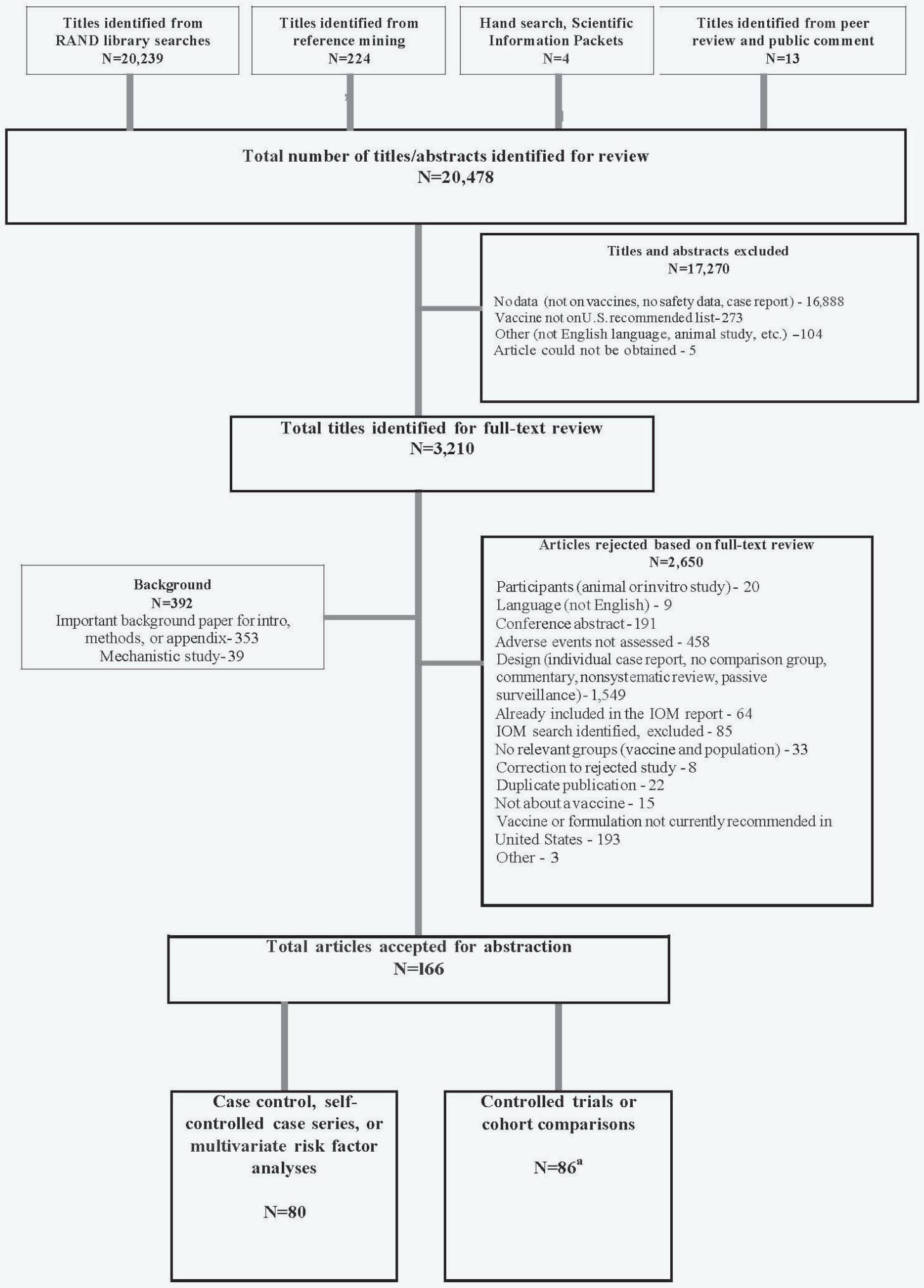

$\mathrm{IOM}=$ Institute of Medicine

aFive studies also contributed multivariate risk factor analyses. 
Table D displays a summary of our results. The second column displays the strength of evidence regarding statistical association of vaccines with AEs. Where we identified no additional studies on a vaccine, our conclusions are based entirely upon the 2011 IOM report. It is important to recognize that the strength of evidence refers to the chances the vaccine is truly associated with a particular $\mathrm{AE}$, rather than the rate of that $\mathrm{AE}$ or severity. Strength of evidence may be high for an $\mathrm{AE}$ that is extremely rare, very mild, or without long-term consequences. Further details on the scientific evidence behind the findings are available in the full report and its appendixes.
Importantly, the vast majority of studies did not report potential risk factors for AEs that were statistically associated with vaccination. Similarly, the severity of AEs was inconsistently reported, as was information that would make independent severity determination possible. 


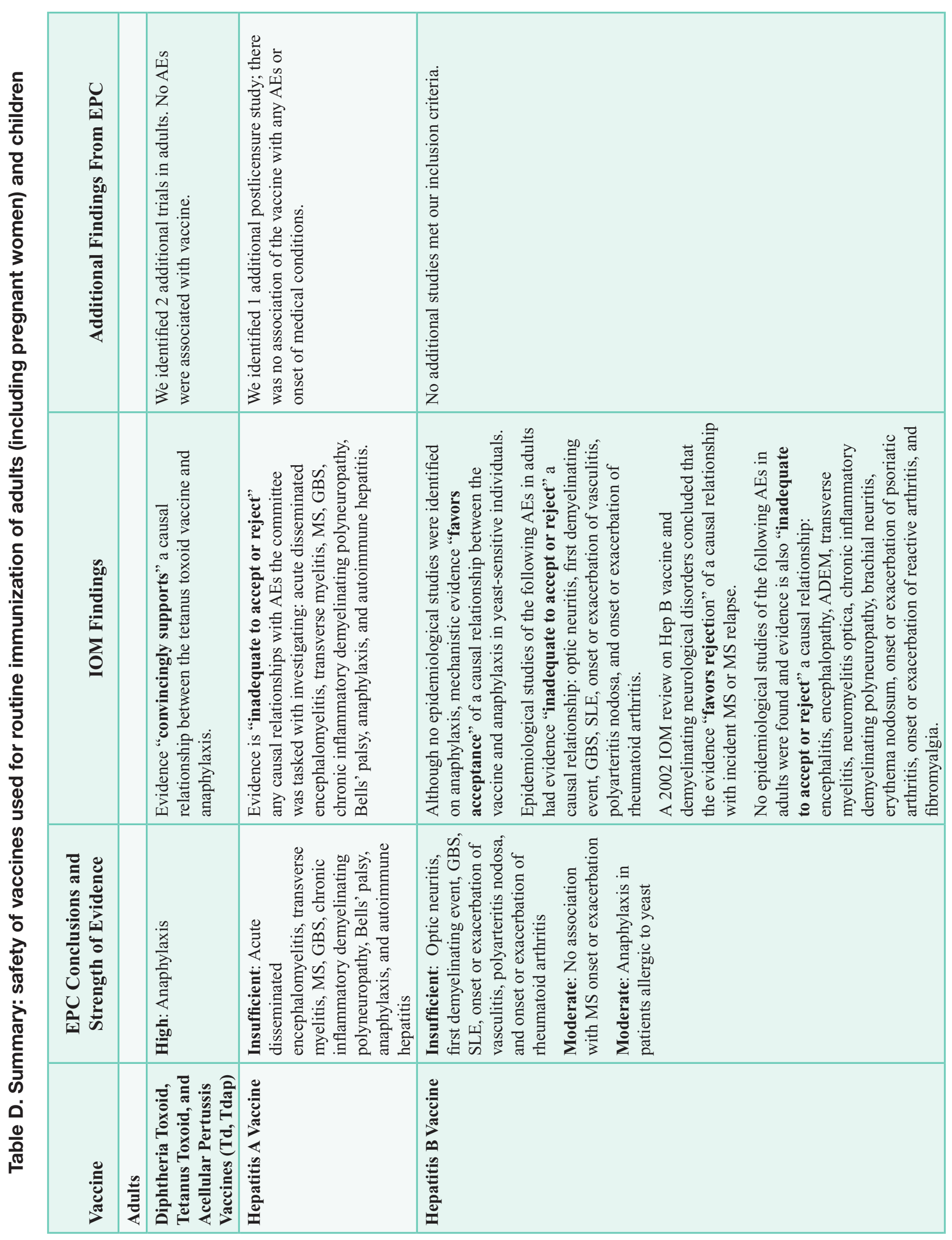




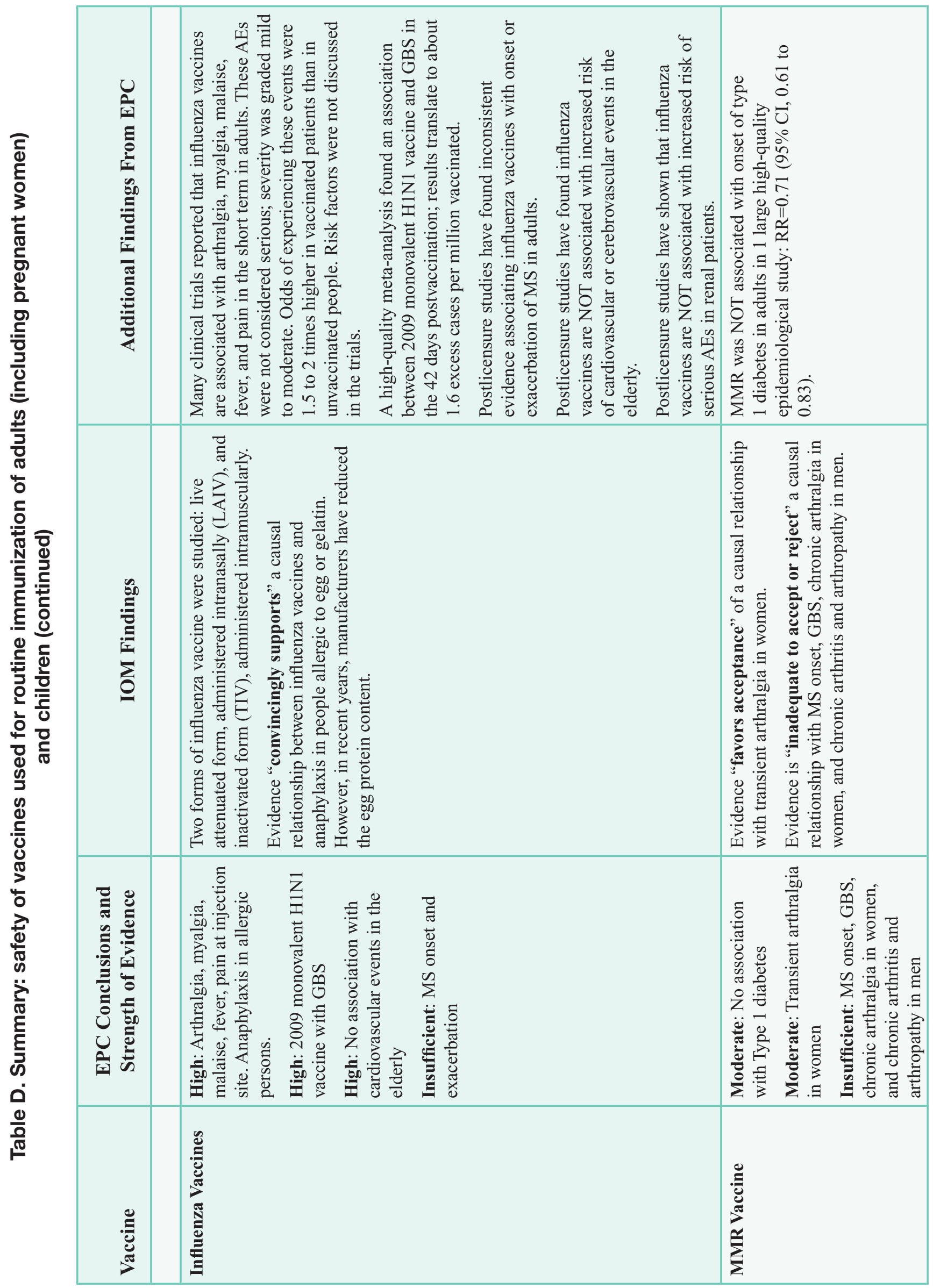




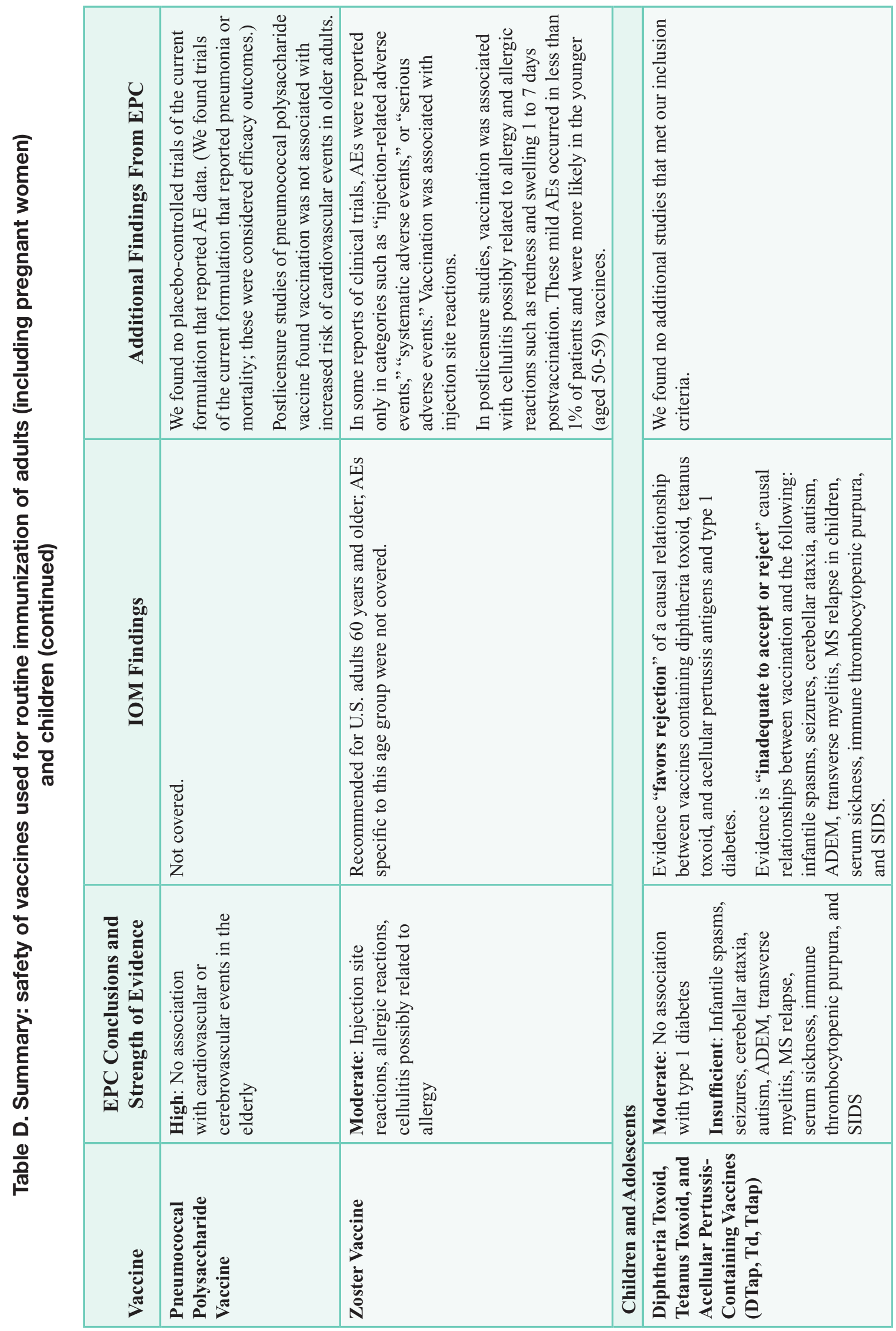




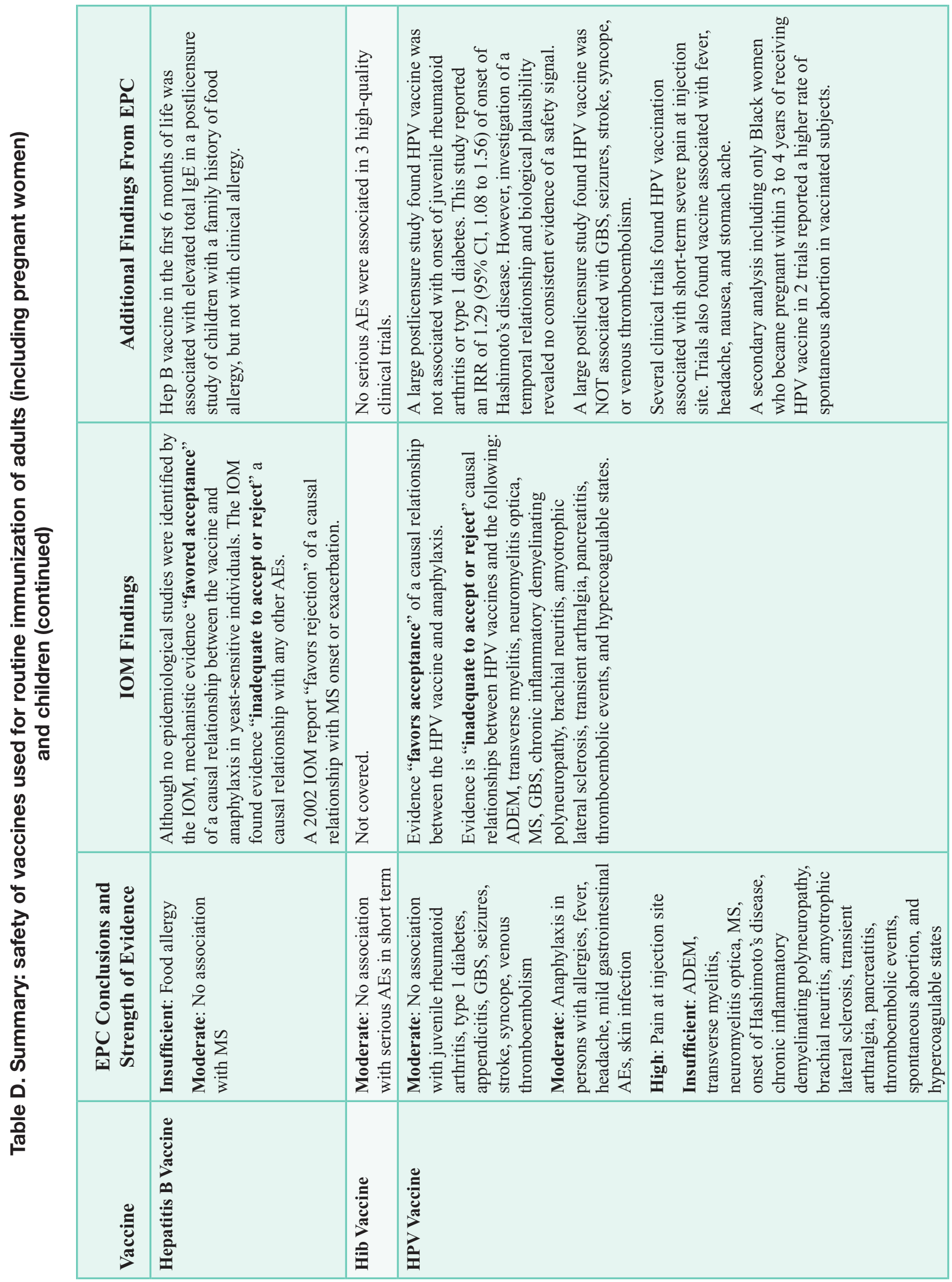




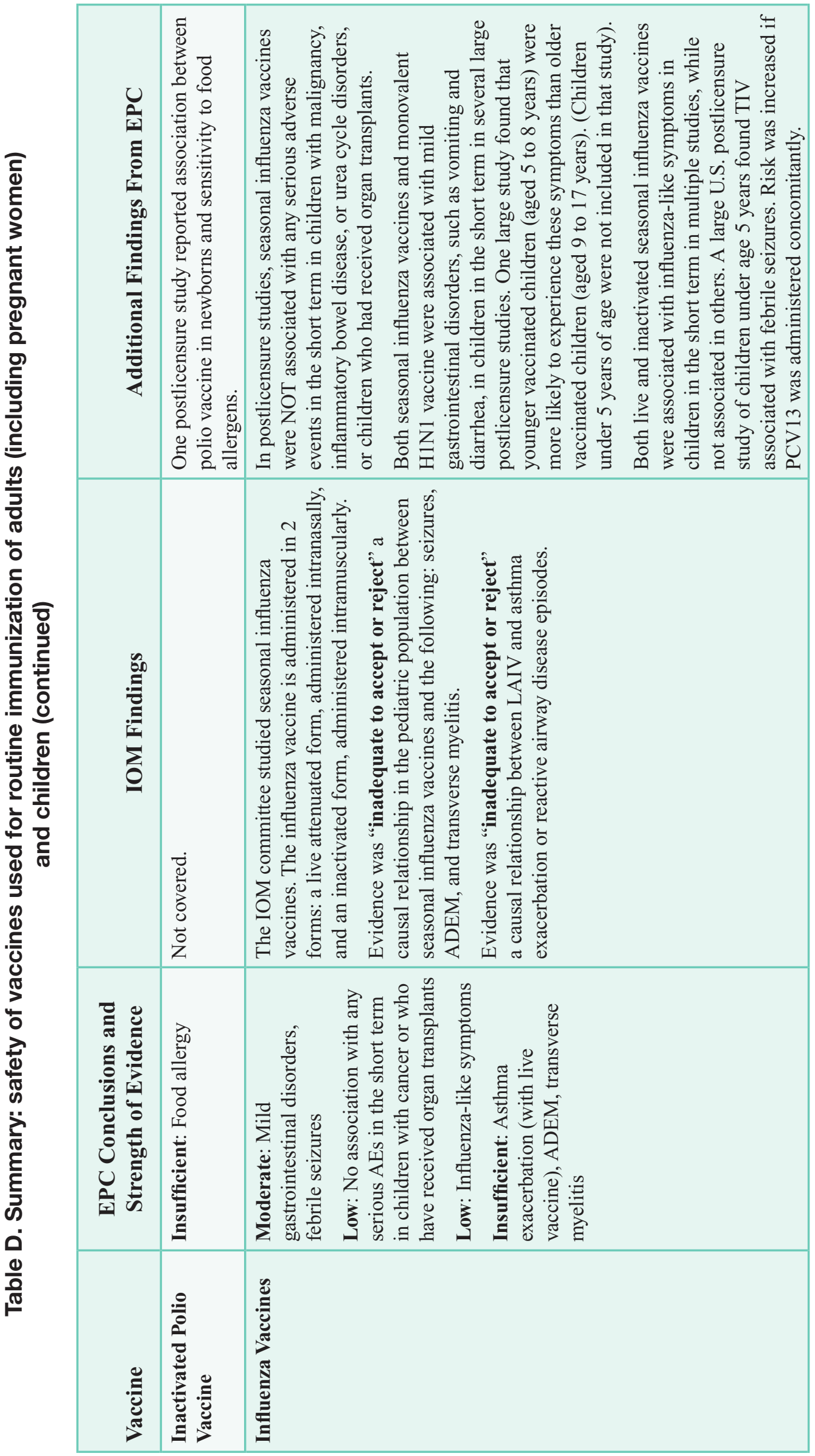




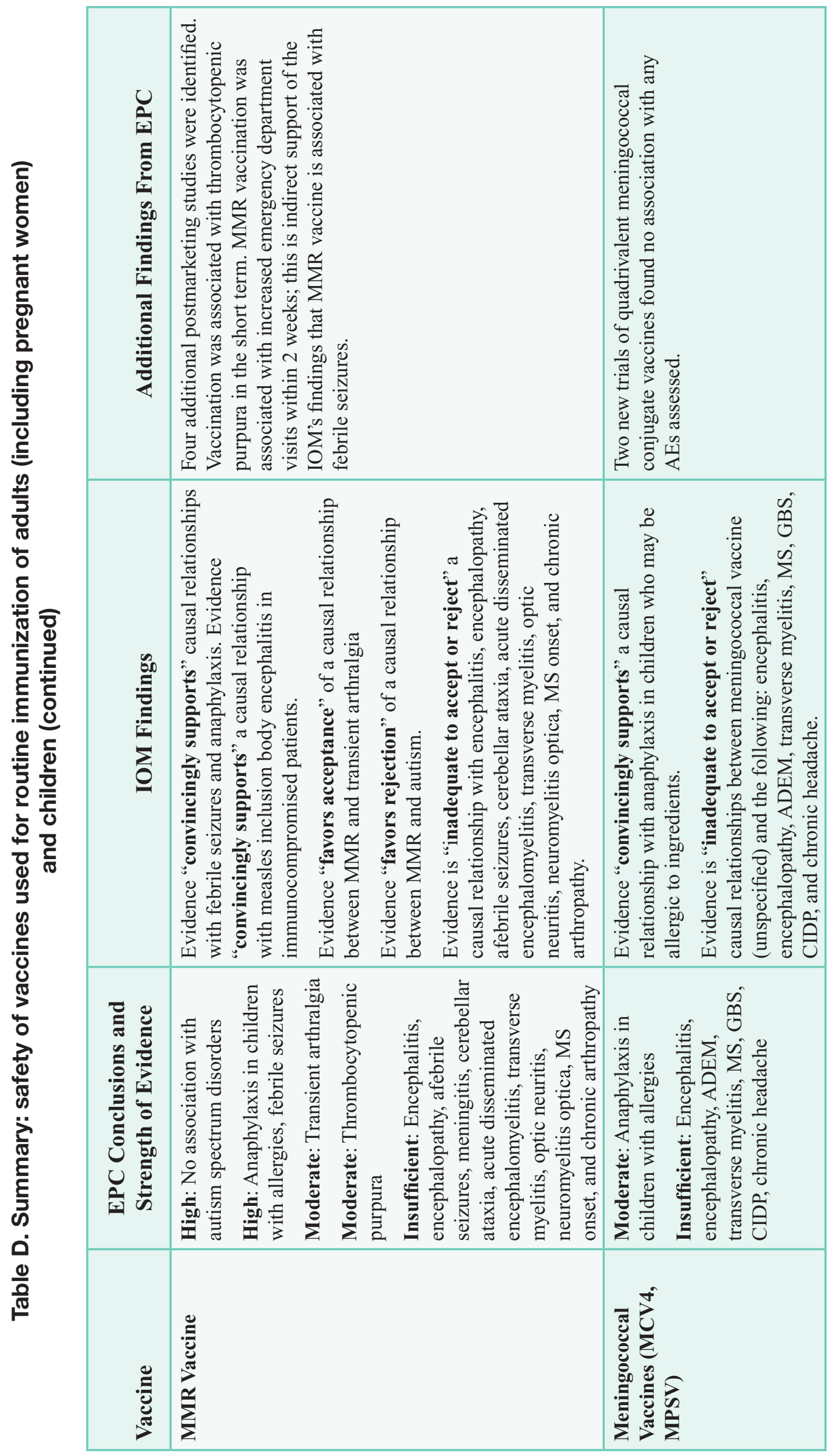




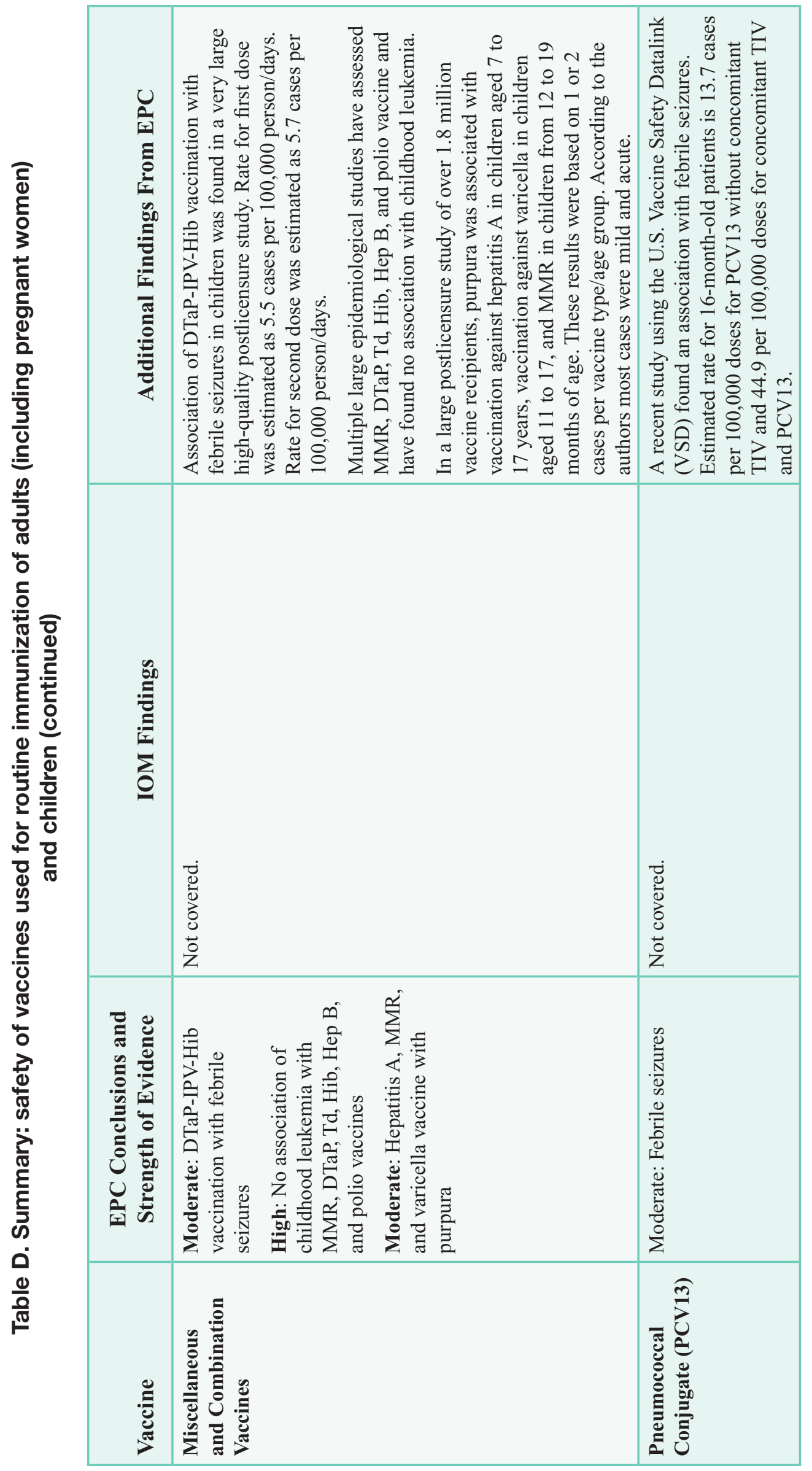




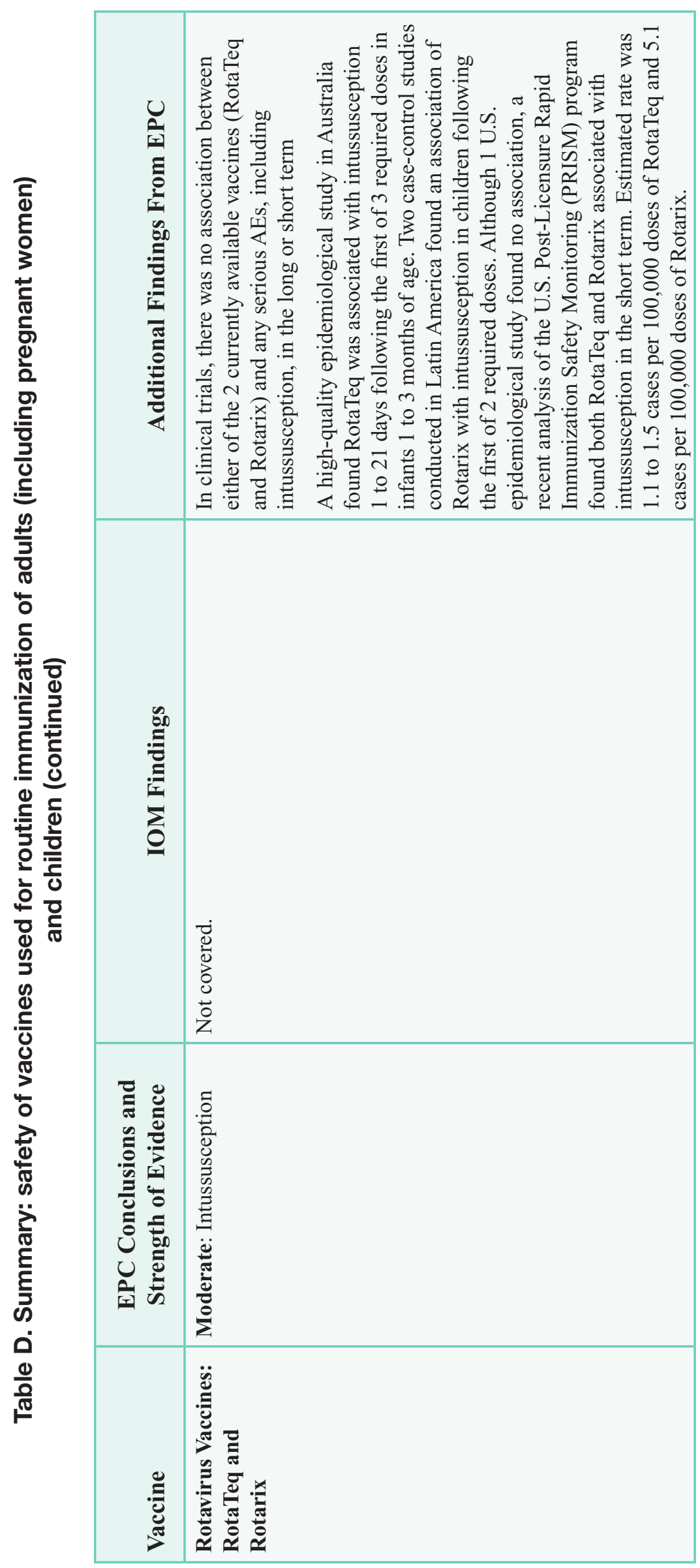




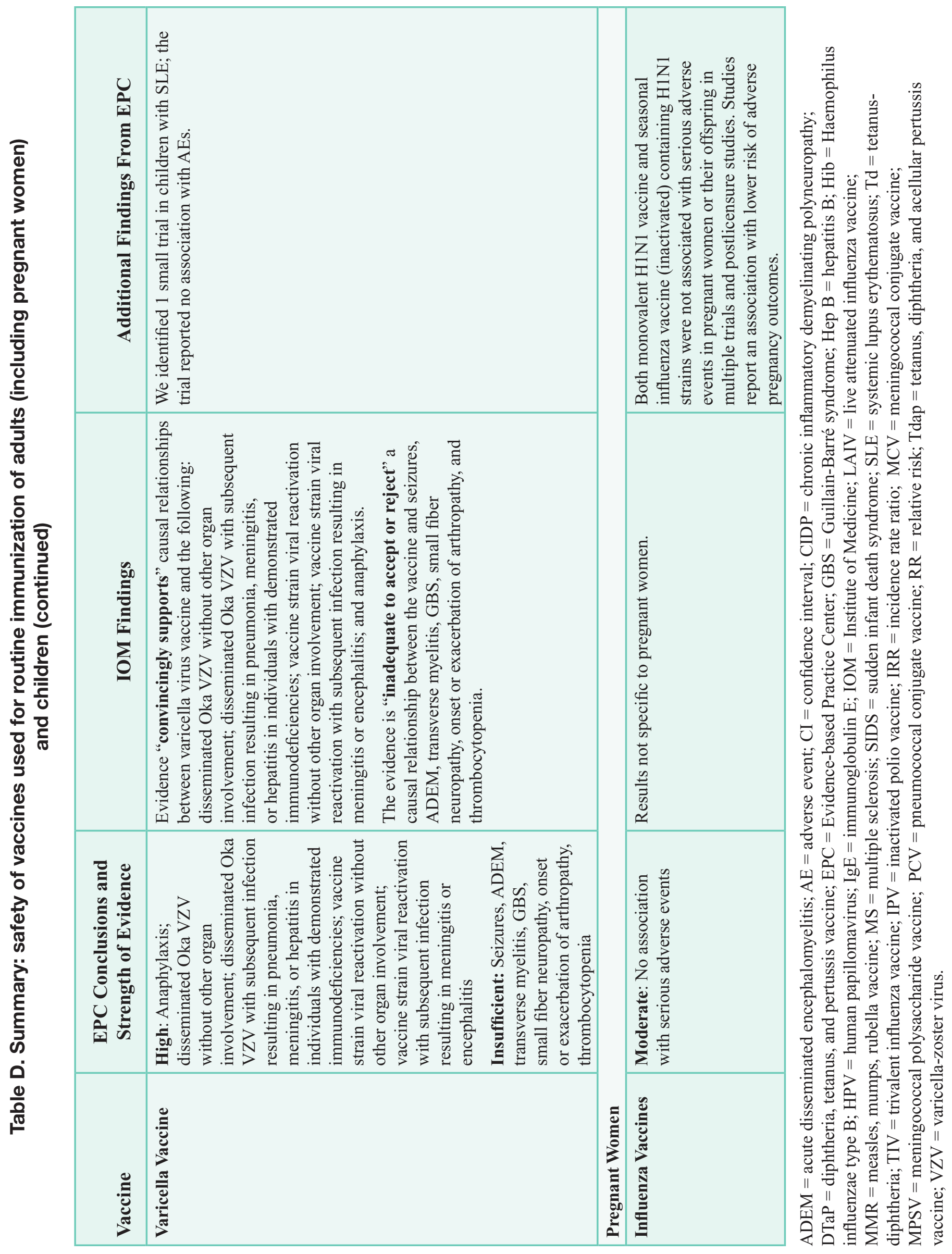




\section{Discussion}

In 2011, the IOM released "Adverse Effects of Vaccines: Evidence and Causality." At the request of AHRQ and $\mathrm{OASH}$, we assessed the additional evidence on the safety of vaccines recommended for routine use among adults, children, and pregnant women as of 2011. We conducted an extensive literature search for clinical trials and observational studies with strong study designs and analysis methods: cohort studies comparing vaccinated and unvaccinated groups, case-control studies, self-controlled case series, and designs using multivariate risk factor analyses. Our results support most findings of the IOM report, add conclusions on some adverse events for which new evidence was identified, and include findings on additional vaccines.

Our findings may allay some patient, caregiver, and health care provider concerns. Strength of evidence is high that vaccines against pneumonia and influenza are not associated with cardiovascular or cerebrovascular events in the elderly; many studies reported a decreased risk for vaccinated patients. Strength of evidence is high that MMR vaccine is not associated with the onset of autism in children; this conclusion supports findings of all previous reviews on the topic. There is moderate-strength evidence that HPV vaccine is not associated with appendicitis, stroke, seizures, syncope, venous thromboembolism, onset of juvenile arthritis, or onset of type 1 diabetes and highstrength evidence that MMR, DTaP (diphtheria, tetanus, and pertussis), Td (tetanus-diphtheria), Hib (Haemophilus influenzae type B), and hepatitis B vaccines are not associated with childhood leukemia.

Evidence of association with vaccines was found for several serious AEs; however, these events were extremely rare. Absolute risk is extremely rare. Strength of evidence is high that 2009 monovalent H1N1 influenza vaccine was associated with Guillain-Barré syndrome (GBS), but results translate to about 1.6 additional cases per million persons vaccinated. Since 2010, U.S. seasonal influenza vaccines have contained an H1N1 strain. No association with GBS has been found for inactivated seasonal vaccine (TIV). Strength of evidence is moderate for association of vaccines against rotavirus with intussusception. Although one U.S. epidemiological study found no association, a recent analysis of the U.S. Post-Licensure Rapid Immunization Safety Monitoring (PRISM) program $^{22}$ found both RotaTeq and Rotarix associated with intussusception in the short term. Estimated rate was 1.1 to 1.5 cases per 100,000 doses of RotaTeq and 5.1 cases per 100,000 doses of Rotarix.

Evidence is insufficient to make conclusions regarding whether several routinely recommended vaccines are associated with serious conditions much as multiple sclerosis (MS), transverse myelitis, and acute disseminated encephalomyelitis (ADEM). This leaves an important research gap.

We identified a recent secondary analysis of data on Black women who participated in two placebo-controlled trials of quadrivalent HPV vaccine (Gardasil) conducted in several countries. In women who became pregnant in the 4 years following the trial, there was a significantly higher rate of spontaneous abortion for women who were vaccinated. ${ }^{23}$ The authors state that the rate of "fetal loss" was statistically similar for vaccinees and nonvaccinees; however, "fetal loss" included planned abortion. In the vaccine group, 19 of 307 pregnancies resulted in spontaneous abortion, compared with 7 of 393 pregnancies in the control group. Databases such as the Vaccine Safety Datalink (VSD) could be used to further investigate this signal in a much larger population.

Conclusions must be viewed in light of the important caveats below.

Literature search procedures were extensive; however, some unpublished trial results may not have been identified. An independent Scientific Resource Center under contract with AHRQ requested Scientific Information Packets from vaccine manufacturers. (The research team was prohibited from contacting manufacturers directly.) Only two companies responded.

We included trials of the formulations currently on the market in the United States. We tried to exclude Phase II studies that used dosages that were never licensed and/ or formulations available only in foreign countries. Some studies reported the potency or formulation of the vaccines in a different manner or unit than reported in the product materials. Large epidemiological studies sometimes included any available formulation of vaccines against a particular disease and did not stratify results by dosage or formulation. For example, the relationship between the "seasonal influenza vaccine" and an AE could be studied over several years of data without addressing the changes in formulation over the seasons.

Our findings are based on only the most rigorous study designs to assess potential statistical associations; however, these designs have limitations that must be considered. Controlled trials often have insufficient sample size to identify very rare AEs and do not have extended followup to identify long-term sequelae. In addition, trials may purposely exclude subjects such as the elderly, pregnant women, and people with medical conditions who could be more susceptible to AEs. For this reason, any comprehensive review of vaccine safety also includes postlicensure studies, but these are not without limitations. 
People who avoid vaccinations (whether purposely or not) may differ from those who receive vaccinations in terms of race, sex, age, socioeconomic status, and preexisting medical conditions, and these differences may be associated with health outcomes. Observational studies attempt to control for such potential confounders by using matched cohorts or multivariate regression analysis; still, some factors such as environmental exposures may be unmeasured or challenging to adequately control for. The self-controlled case series was developed specifically to assess the safety of vaccines; this method eliminates confounding by all time-independent variables by using cases as their own controls and predefined "time windows" before and after vaccination. This design has been used to study purpura, febrile seizures, intussusception, and autism in children. The SCCS assumption of no temporal shifts is difficult to justify in very young children, as any patient characteristics that change with time will not be adequately controlled for.

There may be important AE signals not identified in this report that warrant future research. Passive surveillance systems such as the U.S. Vaccine Adverse Event Reporting System 16 are crucial in identifying signals regarding AEs postlicensure, but they are not designed to assess a statistical association so were excluded from this project. The research gaps below are based on the study designs that met our inclusion criteria.

\section{Research Gaps}

\section{Adults}

There was insufficient evidence to determine whether influenza vaccines are associated with onset or exacerbation of MS.

The unknown association regarding MS and GBS and vaccines for MMR and hepatitis A also presents a research gap; the IOM found evidence inadequate to accept or reject a causal relationship. As these medical conditions are extremely rare, "insufficient" evidence determination may be unavoidable despite additional research.

A recent meta-analysis on 2009 monovalent H1N1 vaccine provided high-strength evidence of association with GBS in adults. As the vaccine is associated with only 1.6 excess cases per million vaccinated, it will be very difficult to assess risk factors.

Some published vaccine trials were not specific in reporting AEs. Broad categories such as "injection-related adverse events," "systemic adverse events," "one or more adverse events," or "serious adverse events" were reported rather than specific AEs. In addition, many studies reported on a list of predefined AEs but did not rate the severity or provide enough information for our investigators to determine severity. Future studies of vaccines should report results with more granularity.

\section{Children and Adolescents}

There is insufficient evidence to determine any potential association between trivalent inactivated vaccine and asthma exacerbation, acute disseminated encephalomyelitis, and transverse myelitis.

Febrile seizures were associated with MMR, influenza, and pneumococcal conjugate vaccines. Younger age was associated with increased risk in several studies. Largescale epidemiological studies could determine other patient risk factors.

A large U.S. postlicensure study found associations between both Rotarix and RotaTeq and intussusception in the short term following vaccination; patient risk factors were not reported.

Strong evidence for a lack of association of HPV vaccines with several serious medical conditions (juvenile rheumatoid arthritis, type 1 diabetes, GBS) has been found in large postlicensure studies. However, there is insufficient evidence regarding other serious conditions such as MS, chronic inflammatory demyelinating polyneuropathy, amyotrophic lateral sclerosis, and pancreatitis. Importantly, a recent analysis of long-term followup data from Black women enrolled in two trials of Gardasil showed a possible increased risk of miscarriage of pregnancies within 4 years of vaccination. Large datasets from U.S. managed care organizations include medical records on both immunization and pregnancy, so they could be used to investigate any potential association.

There is insufficient evidence to determine the possible association, if any, between vaccines such as DTaP, meningococcal vaccine, and varicella vaccine and the onset of nervous system conditions such as ADEM, transverse myelitis, MS, and GBS. Large-scale epidemiological studies could provide additional data; however, as these medical conditions are extremely rare, it may not be possible to reach a level of evidence beyond "insufficient."

\section{Pregnant Women}

There is moderate strength of evidence that inactivated influenza vaccine is not associated with serious adverse events in pregnant women or their offspring. Given the 2013 recommendation to administer the Tdap vaccine during every pregnancy, passive surveillance systems should be monitored regularly for AEs in this population.

A reliable system of tracking when in pregnancy the vaccine was given is extremely important. In addition, in any study of vaccines and pregnancy, followup of newborns 
should be sufficiently long, as not all adverse effects may be apparent immediately after birth. The need for large numbers of pregnant exposures is particularly important given the relatively low frequency of some birth defects and the need to define which are associated with vaccine. In addition, little is known about the patient factors that may influence the effect of the vaccine. Another unknown is how vaccination in pregnancy may affect the newborn's immune system/reaction to newborn vaccinations. The Vaccines and Medications in Pregnancy Surveillance System (VAMPSS) has components to monitor safety of maternally administered vaccines and their effect on recipients and their offspring. VAMPSS is a collaboration of the American Academy of Allergy, Asthma \& Immunology; the Organization of Teratology Information Specialists (OTIS) Research Center at the University of California San Diego; and the Slone Epidemiology Center (SEC) at Boston University. The U.S. Department of Health and Human Services Biomedical Advanced Research and Development Authority contracted VAMPSS to perform safety monitoring of H1N1 influenza vaccine administered during the 2009 pandemic. As this report was being finalized, a VAMPSS study on the safety of H1N1 was released;24,25 the authors found no meaningful evidence of increased risk of major birth defects, miscarriage, or low birth weight for gestational age. Existing Federal systems such as the VSD and/or PRISM could potentially be used as well.

\section{General Methodological Observations}

Advanced health information technology systems that contain both vaccination and health outcome records can be used to conduct high-quality epidemiological studies. In the United States, the VSD contains data obtained through such systems at nine very large managed care organizations. The FDA's Mini-Sentinel program PRISM system also conducts active surveillance using electronic health care databases from managed care organizations. Nations with singlepayer health care systems often have electronic registries that allow very large epidemiological studies of entire populations. Studies using these databases have greater validity than studies that rely on surveys that use patient/ parent recall for ascertainment of vaccination or health outcome. Not only are such surveys subject to recall bias, but there may be no way of determining the formulation or brand of vaccination.

Observational studies should be powered adequately to determine risk factors such as demographic and health characteristics of patients. Analysis should be stratified by formulation and brand of vaccine, if possible. This is especially true for influenza vaccine, which differs from season to season.
Independent abstraction and systematic reassessment of the studies included in the IOM consensus report "Adverse Effects of Vaccines: Evidence and Causality" may be a useful future endeavor. Odds ratios could be calculated for each event reported in each trial and postlicensure study and, where appropriate, meta-analysis conducted to calculate overall odds ratios for each $\mathrm{AE}$ and each vaccine type. If the additional studies were abstracted, the totality of data abstracted could be statistically analyzed to explore additional hypotheses and issues beyond the scope of the current report.

\section{References}

1. Ten great public health achievements--United States, 1900-1999. MMWR Morb Mortal Wkly Rep. 1999 Apr 2;48(12):241-3. PMID: 10220250.

2. National, state, and local area vaccination coverage among children aged 19-35 months - United States, 2011. MMWR Morb Mortal Wkly Rep. 2012 Sep 7;61:689-96. PMID: 22951450.

3. National and state vaccination coverage among adolescents aged 13-17 years - United States, 2011. MMWR Morb Mortal Wkly Rep. 2012 Aug 31;61:671-7. PMID: 22932301.

4. Adult vaccination coverage--United States, 2010. MMWR Morb Mortal Wkly Rep. 2012 Feb 3;61(4):66-72. PMID: 22298302.

5. Influenza vaccination coverage among pregnant women-United States, 2010-11 influenza season. MMWR Morb Mortal Wkly Rep. 2011 Aug 19;60(32):1078-82. PMID: 21849964.

6. Wakefield AJ, Murch SH, Anthony A, et al. Ileal-lymphoidnodular hyperplasia, non-specific colitis, and pervasive developmental disorder in children. Lancet. $1998 \mathrm{Feb}$ 28;351(9103):637-41. PMID: 9500320.

7. Retraction--Ileal-lymphoid-nodular hyperplasia, non-specific colitis, and pervasive developmental disorder in children. Lancet. 2010 Feb 6;375(9713):445. PMID: 20137807.

8. Freed GL, Clark SJ, Hibbs BF, et al. Parental vaccine safety concerns. The experiences of pediatricians and family physicians. Am J Prev Med. 2004 Jan;26(1):11-4. PMID: 14700706.

9. Advisory Committee on Immunization Practices. Recommended adult immunization schedule: United States, 2011. Ann Intern Med. 2011;154(154):168-73.

10. Centers for Disease Control and Prevention. ACIP Recommendations Advisory Committee for Immunization Practices (ACIP). www.cdc.gov/vaccines/pubs/acip-list.htm. Accessed April 2012.

11. Centers for Disease Control and Prevention. Guidelines for Vaccinating Pregnant Women. www.cdc.gov/vaccines/pubs/ preg-guide.htm. Accessed April 2012. 
12. Institute of Medicine. Adverse Effects of Vaccines: Evidence and Causality. Washington, DC: The National Academy Press; 2011.

13. Higgins JPT, Green S, eds. Cochrane Handbook for Systematic Reviews of Interventions Version 5.1.0 [updated March 2011]. The Cochrane Collaboration; 2011. www.cochrane-handbook.org.

14. Mann CJ. Observational research methods. Research design II: cohort, cross sectional, and case-control studies. Emerg Med J. 2003 Jan;20(1):54-60. PMID: 12533370.

15. Whitaker HJ, Farrington CP, Spiessens B, et al. Tutorial in biostatistics: the self-controlled case series method. Stat Med. 2006 May 30;25(10):1768-97. PMID: 16220518.

16. Haber $\mathrm{P}$, Iskander J, Walton $\mathrm{K}$, et al. Internet-based reporting to the Vaccine Adverse Event Reporting System: a more timely and complete way for providers to support vaccine safety. Pediatrics. 2011 May;127(Suppl 1):S39-S44. PMID: 21502243.

17. Institute of Medicine. The Childhood Immunization Schedule and Safety: Stakeholder Concerns, Scientific Evidence, and Future Studies. Washington, DC: The National Academies Press; 2013.

18. National Institutes of Health, National Cancer Institute. Common Terminology Criteria for Adverse Events (CTCAE). Version 4.0. May 28, 2009.

19. Santaguida PL, Raina P. The Development of the McHarm Quality Assessment Scale for Adverse Events: Delphi Consensus on Important Criteria for Evaluating Harms. 2008. http://hiru.mcmaster.ca/epc/mcharm.pdf. Downloaded December 2008.

20 Owens DK, Lohr KN, Atkins D, et al. AHRQ series paper 5: grading the strength of a body of evidence when comparing medical interventions--Agency for Healthcare Research and Quality and the effective health-care program. J Clin Epidemiol. 2010 May;63(5):513-23. PMID: 19595577.

21. Balshem H, Helfand M, Schunemann HJ, et al. GRADE guidelines: 3. Rating the quality of evidence. J Clin Epidemiol. 2011 Apr;64(4):401-6. PMID: 21208779.

22. Yih K, Lieu T, Kulldorff M, et al. Intussusception Risk After Rotavirus Vaccination in U.S. Infants. Mini-Sentinel Coordinating Center; June 2013. www.mini-sentinel.org/ work_products/PRISM/Mini-Sentinel_PRISM_Rotavirusand-intussusception-Report.pdf. Accessed June 2013.

23. Clark LR, Myers ER, Huh W, et al. Clinical trial experience with prophylactic human papillomavirus $6 / 11 / 16 / 18$ vaccine in young black women. J Adolesc Health.

2013 Mar;52(3):322-9. PMID: 23299013.

24. Louik C, Ahrens K, Kerr S, et al. Risks and safety of pandemic H1N1 influenza vaccine in pregnancy: exposure prevalence, preterm delivery, and specific birth defects. Vaccine. 2013 Oct 17;31(44):5033-40. PMID: 24016804.
25. Chambers CD, Johnson D, Xu R, et al. Risks and safety of pandemic h1n1 influenza vaccine in pregnancy: birth defects, spontaneous abortion, preterm delivery, and small for gestational age infants. Vaccine. 2013 Oct 17;31(44):502632. PMID: 24016809.

\section{Addendum}

This evidence report summarizes scientific data on the safety of vaccines currently recommended in the United States. High strength of evidence was found for an association of measles-mumps-rubella (MMR) vaccine with febrile seizures in children under age 5 (Key Question 2c). On May 19, 2014 (while this evidence report was in press), an important study investigating the timing of MMR and MMRV (measles-mumps-rubella-varicella) vaccine and risk of febrile seizures 1 was published. We felt this information warranted inclusion in this review of the evidence.

Researchers conducted a self-controlled case series analysis using records from over 300,000 U.S. children from the Vaccine Safety Datalink (VSD). They found timing was unrelated to risk of post-vaccination seizure in infants, but found that delaying measles containing vaccines past 15 months results in a higher risk of seizures.

According to the study's authors, these adverse events are very rare, and occurred at the rate of about 1 per 100,000 person-days at seven months of age, compared to a high of five per 100,000 person-days at 17 months of age.

\section{Reference}

1. Hambidge S J, Newcomer SR, Narwaney KJ, et al. Timely versus delayed early childhood vaccination and seizures. Pediatrics. 2014 Jun;133(6):e1492-e1499. PMID: 24843064.

\section{Full Report}

This executive summary is part of the following document: Maglione MA, Gidengil C, Das L, Raaen L, Smith A, Chari R, Newberry S, Hempel S, Shanman R, Perry T, Goetz MB. Safety of Vaccines Used for Routine Immunization in the United States. Evidence Report/Technology Assessment No. 215. (Prepared by the Southern California Evidencebased Practice Center under Contract No. 290-2007-10062I.) AHRQ Publication No. 14-E002-EF. Rockville, MD: Agency for Healthcare Research and Quality; July 2014. www.effectivehealthcare.ahrq.gov/reports/final.cfm. DOI: https://doi.org/10.23970/AHRQEPCERTA215.

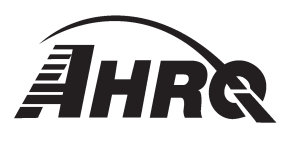

AHRQ Pub. No. 14-E002-1-EF July 2014 
